\title{
International investment position
}




\title{
8.1 International investment position \\ 8. Summary table
}

\author{
Balance sheets valued at end of year
}

$£$ billion

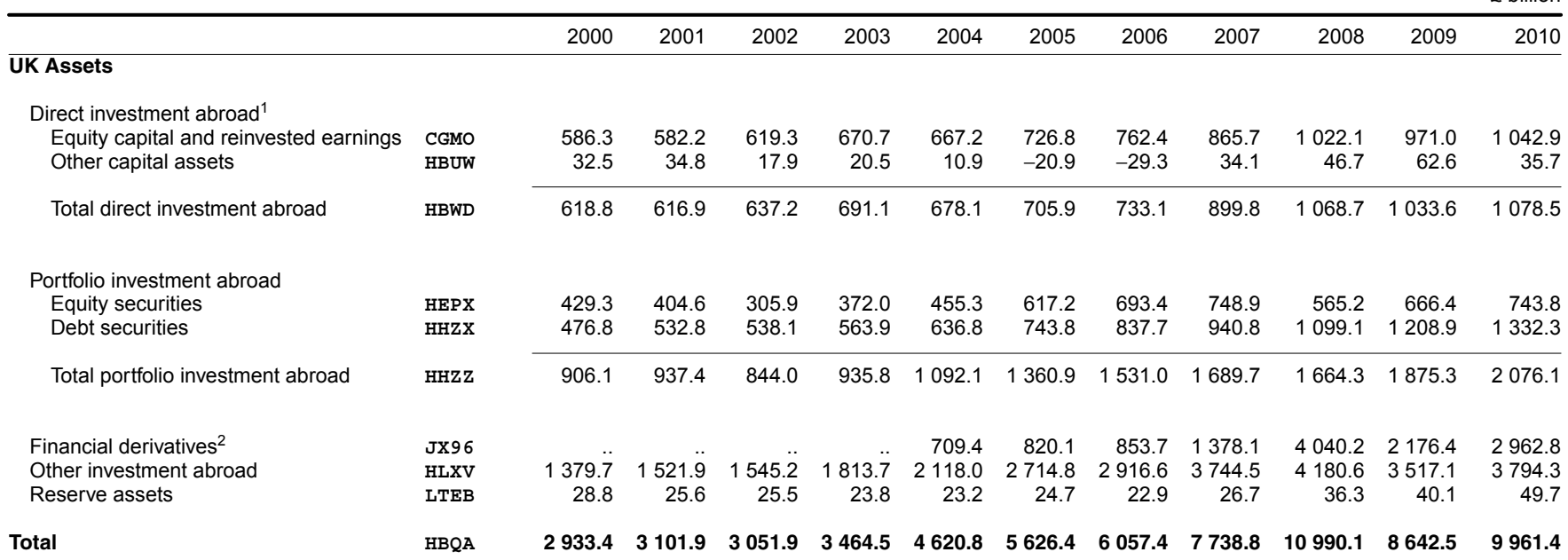

\section{UK Liabilities}

Direct investment in the UK ${ }^{1}$
Equity capital and reinvested earnings
Other capital liabilities
Total direct investment in the UK
Portfolio investment in the UK
Equity securities
Debt securities
Total portfolio investment in the UK
$\begin{aligned} & \text { Financial derivatives } \\ & \end{aligned}$
Other investment in the UK
Total

\begin{tabular}{|c|c|c|c|c|c|c|c|c|c|c|}
\hline 240.6 & 259.7 & 229.2 & 245.7 & 267.9 & 368.7 & 433.1 & 495.8 & 545.7 & 524.7 & $\begin{array}{l}570.7 \\
122.3\end{array}$ \\
\hline 69.8 & 103.7 & 111.4 & 109.9 & 115.5 & 125.5 & 144.2 & 118.0 & 114.7 & 127.6 & \\
\hline 310.4 & 363.5 & 340.6 & 355.5 & 383.3 & 494.2 & 577.3 & 613.8 & 660.4 & 652.3 & 692.9 \\
\hline 673.7 & 584.1 & 442.7 & 527.1 & 574.7 & 659.3 & 780.8 & 835.8 & 605.5 & 853.6 & 944.6 \\
\hline 393.8 & 429.1 & 482.6 & 555.8 & 653.2 & 802.4 & 922.6 & 1109.8 & 1374.7 & 1551.9 & 1578.5 \\
\hline 1067.6 & 1013.2 & 925.3 & 1082.9 & 1227.9 & 1461.7 & 1703.5 & 1945.7 & 1980.2 & 2405.5 & 2523.1 \\
\hline & & & & 715.0 & 831.1 & 890.5 & 1392.2 & 3915.3 & 2096.8 & 2895.0 \\
\hline 1651.6 & 1861.9 & 1898.0 & 2135.2 & 2511.8 & 3109.0 & 3270.0 & 4115.6 & 4518.1 & 3792.0 & 4047.6 \\
\hline 3029.5 & 3238.5 & 3163.9 & 3573.6 & 4838.1 & 5896.0 & 6441.2 & 8067.3 & 11073.9 & 8946.6 & 10158.6 \\
\hline
\end{tabular}

Net International Investment Position

\section{Direct investment ${ }^{1}$ Other capital \\ Total net direct investment \\ Portfolio investment Equity securities Debt securities \\ Total net portfolio investment \\ Financial derivatives ${ }^{2}$ \\ Other investment \\ Reserve assets}

Equity capital and reinvested earnings HBSH CGKF HBWQ

CGNE CGNF CGNH

JX98 CGNG LTEB
HBSH

\begin{tabular}{rrrrrrrrrrr}
345.7 & 322.4 & 390.1 & 425.0 & 399.3 & 358.1 & 329.3 & 369.8 & 476.4 & 446.4 & 472.2 \\
-37.3 & -69.0 & -93.5 & -89.4 & -104.6 & -146.4 & -173.5 & -83.9 & -68.0 & -65.0 & -86.6 \\
\hline 308.4 & 253.5 & 296.6 & 335.6 & 294.7 & 211.7 & 155.8 & 285.9 & 408.4 & 381.3 & 385.6
\end{tabular}

\begin{tabular}{rrrrrrrrrrr}
-244.4 & -179.4 & -136.8 & -155.1 & -119.4 & -42.1 & -87.5 & -86.9 & -40.3 & -187.2 & -200.8 \\
82.9 & 103.7 & 55.5 & 8.1 & -16.4 & -58.6 & -84.9 & -169.0 & -275.6 & -343.0 & -246.2 \\
\hline-161.5 & -75.7 & -81.3 & -147.0 & -135.8 & -100.8 & -172.4 & -255.9 & -315.9 & -530.2 & -447.0
\end{tabular}
HBQC

$\begin{array}{rrrrrrrrrrr}. \ddot{2} & . . . & . . & . . & -5.6 & -11.0 & -36.8 & -14.1 & 124.9 & 79.6 & 67.8 \\ -271.9 & -339.9 & -352.8 & -321.5 & -393.9 & -394.2 & -353.4 & -371.0 & -337.5 & -274.9 & -253.4 \\ 28.8 & 25.6 & 25.5 & 23.8 & 23.2 & 24.7 & 22.9 & 26.7 & 36.3 & 40.1 & 49.7 \\ & & & & & & & & & & \\ \mathbf{- 9 6 . 2} & -\mathbf{1 3 6 . 5} & \mathbf{- 1 1 2 . 0} & -\mathbf{1 0 9 . 2} & -\mathbf{2 1 7 . 3} & \mathbf{- 2 6 9 . 6} & \mathbf{- 3 8 3 . 8} & \mathbf{- 3 2 8 . 4} & \mathbf{- 8 3 . 8} & \mathbf{- 3 0 4 . 1} & \mathbf{- 1 9 7 . 3}\end{array}$

$\begin{array}{lllllllllll}-96.2 & -136.5 & -112.0 & -109.2 & -217.3 & -269.6 & -383.8 & -328.4 & -83.8 & -304.1 & -197.3\end{array}$

$1.6 \quad 1.6 \quad 1.5 \quad 1.6$

$\begin{array}{lllll}1.5 & 1.5 & 2.0 & 1.9 & 1.9\end{array}$

\footnotetext{
1 The latest year's data for foreign direct investment are provisional as these figures are subject to annual benchmarking.

2 Data from 2004 cover the assets and liabilities of UK banks. From 2010, the

assets and liabilities of UK securities dealers are also included.
} 


\subsection{International investment position}

8.2 Sector analysis

Balance sheets valued at end of year

$£$ billion

\begin{tabular}{|c|c|c|c|c|c|c|c|c|c|c|c|c|}
\hline & & 2000 & 2001 & 2002 & 2003 & 2004 & 2005 & 2006 & 2007 & 2008 & 2009 & 2010 \\
\hline \multicolumn{13}{|l|}{ UK Assets } \\
\hline Monetary financial institutions & CGNJ & 1421.8 & 1535.0 & 1572.6 & 1737.9 & 2700.6 & 3248.3 & 3530.4 & 4838.5 & 7991.8 & 5556.7 & 5786.4 \\
\hline Central government & CGNK & 37.7 & 35.2 & 36.4 & 34.2 & 33.4 & 35.9 & 35.2 & 42.5 & 55.4 & 63.6 & 75.1 \\
\hline Public corporations & CGNL & 4.6 & 3.8 & 4.2 & 4.2 & 4.7 & 3.4 & 1.7 & 1.7 & 1.6 & 1.2 & 1.2 \\
\hline Other sectors & CGNM & 1469.3 & 1528.0 & 1438.6 & 1688.1 & 1882.1 & 2338.8 & 2490.1 & 2856.0 & 2941.4 & 3021.1 & 4098.6 \\
\hline Total & HBQA & 2933.4 & 3101.9 & 3051.9 & 3464.5 & 4620.8 & 5626.4 & 6057.4 & 7738.8 & 10990.1 & 8642.5 & 9961.4 \\
\hline \multicolumn{13}{|l|}{ UK Liabilities } \\
\hline Monetary financial institutions & HBYJ & 1486.6 & 1623.8 & 1707.1 & 1893.3 & 2895.0 & 3413.5 & 3707.5 & 5082.9 & 8137.0 & 5802.6 & 5931.4 \\
\hline Central government & CGOG & 65.6 & 63.3 & 59.2 & 70.9 & 89.3 & 115.2 & 141.2 & 169.6 & 226.7 & 246.1 & 340.6 \\
\hline Local authorities & $\mathrm{CGOH}$ & 0.8 & 0.8 & 0.9 & 1.1 & 1.7 & 1.9 & 2.1 & 2.1 & 2.5 & 3.0 & 3.2 \\
\hline Public corporations & CGOI & - & - & - & - & 0.5 & 0.4 & 0.4 & 0.4 & 0.4 & 0.4 & 0.4 \\
\hline Other sectors & HCON & 1476.6 & 1550.5 & 1396.7 & 1608.3 & 1851.6 & 2365.0 & 2590.0 & 2812.3 & 2707.3 & 2894.4 & 3883.0 \\
\hline Total & $\mathrm{HBQB}$ & 3029.5 & 3238.5 & 3163.9 & 3573.6 & 4838.1 & 5896.0 & 6441.2 & 8067.3 & 11073.9 & 8946.6 & 10158.6 \\
\hline \multicolumn{13}{|c|}{ Net International Investment Position } \\
\hline Monetary financial institutions & HDIJ & -64.8 & -88.8 & -134.5 & -155.4 & -194.4 & -165.1 & -177.0 & -244.4 & -145.3 & -246.0 & -145.0 \\
\hline Central government & CGOK & -27.9 & -28.2 & -22.8 & -36.7 & -55.9 & -79.3 & -106.0 & -127.0 & -171.3 & -182.5 & -265.5 \\
\hline Local authorities & $-\mathrm{CGOH}$ & -0.8 & -0.8 & -0.9 & -1.1 & -1.7 & -1.9 & -2.1 & -2.1 & -2.5 & -3.0 & -3.2 \\
\hline Public corporations & CGOL & 4.6 & 3.8 & 4.2 & 4.2 & 4.2 & 2.9 & 1.3 & 1.3 & 1.2 & 0.8 & 0.8 \\
\hline Other sectors & HDKB & -7.3 & -22.5 & 41.9 & 79.8 & 30.5 & -26.3 & -100.0 & 43.7 & 234.1 & 126.6 & 215.6 \\
\hline Total & $\mathrm{HBQC}$ & -96.2 & -136.5 & -112.0 & -109.2 & -217.3 & -269.6 & -383.8 & -328.4 & -83.8 & -304.1 & -197.3 \\
\hline
\end{tabular}




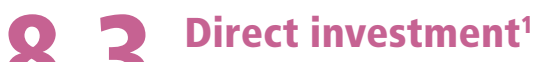

Balance sheets valued at end of year

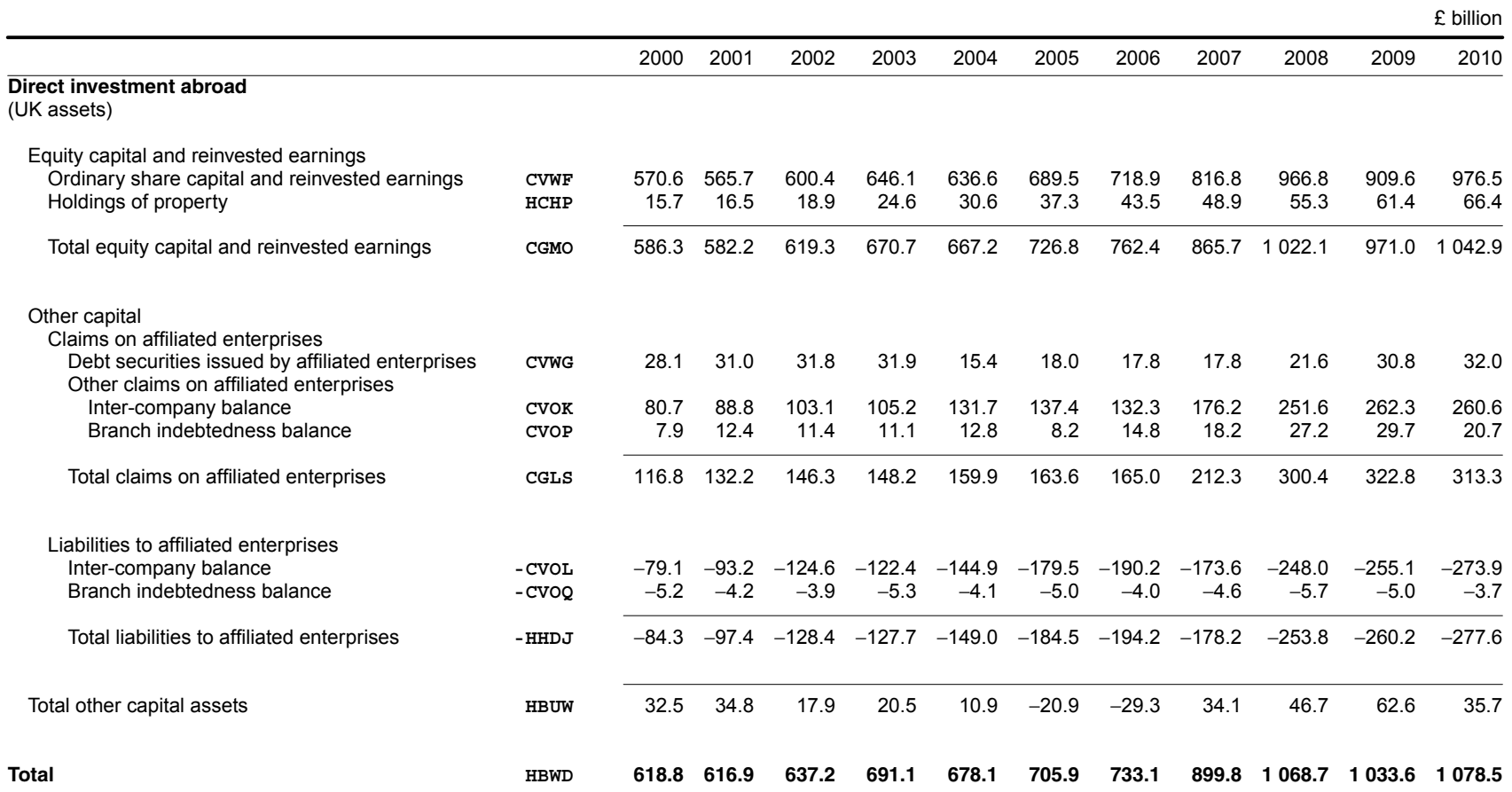

Direct investment in the UK

(UK liabilities)

Equity capital and reinvested earnings

Share capital and reinvested earnings

Quoted share capital and reinvested earnings ${ }^{2}$

Unquoted share capital and reinvested earnings

Total share capital and reinvested earnings

Holdings of UK property

Total equity capital and reinvested earnings

CVVB

CVVC

HBUX

HCQM

HBUY

Other capital

Liabilities to direct investors

Debt securities issued by affiliated enterprises

Other liabilities to direct investors

Inter-company balance

Branch indebtedness balance

Total liabilities to direct investors

Claims on direct investors

Inter-company balance

Branch indebtedness balance

Total claims on direct investors

Total other capital liabilities

Total

\begin{tabular}{lrrrrrrrrrrr} 
CVVD & 11.0 & 17.5 & 17.3 & 16.0 & 20.4 & 28.3 & 38.6 & 33.1 & 40.2 & 43.6 & 42.3 \\
& & & & & & & & & & & \\
CVVJ & 103.8 & 133.1 & 147.0 & 142.2 & 147.6 & 165.7 & 166.6 & 150.4 & 160.1 & 144.2 & 143.7 \\
CVVM & 8.5 & 9.4 & 8.7 & 10.7 & 8.5 & 8.5 & 16.0 & 9.9 & 11.7 & 17.5 & 17.9 \\
\cline { 2 - 10 } HBVB & 123.2 & 160.0 & 173.0 & 168.9 & 176.5 & 202.5 & 221.2 & 193.5 & 212.0 & 205.4 & 203.9
\end{tabular}

- CVVI

CVVL

-HBVA

HBVC

HBWI

\begin{tabular}{rrrrrrrrrrr}
$-\overline{2}$ & - & 20.7 & 25.8 & 35.6 & 73.6 & 38.9 & 32.9 & 30.8 & 14.5 & 37.9 \\
& 245.6 & 192.6 & 204.0 & 214.5 & 276.8 & 374.0 & 438.1 & 490.9 & 485.6 & 506.2 \\
\hline 227.1 & 245.6 & 213.3 & 229.8 & 250.1 & 350.4 & 412.9 & 471.0 & 521.7 & 500.1 & 544.1 \\
13.5 & 14.1 & 15.9 & 15.9 & 17.8 & 18.4 & 20.2 & 24.8 & 24.0 & 24.5 & 26.5 \\
& & & & & & & & & & \\
\hline 240.6 & 259.7 & 229.2 & 245.7 & 267.9 & 368.7 & 433.1 & 495.8 & 545.7 & 524.7 & 570.7
\end{tabular}

$\begin{array}{lllllllllll}-51.3 & -53.4 & -60.5 & -55.6 & -58.6 & -72.7 & -73.0 & -74.8 & -94.8 & -77.0 & -80.4\end{array}$

\begin{tabular}{rrrrrrrrrrr}
-2.1 & -2.9 & -1.1 & -3.5 & -2.4 & -4.3 & -3.9 & -0.7 & -2.5 & -0.8 & -1.2 \\
\hline-53.4 & -56.3 & -61.6 & -59.0 & -61.0 & -77.0 & -76.9 & -75.5 & -97.3 & -77.7 & -81.6
\end{tabular}

\begin{tabular}{lllllllllll}
\hline 69.8 & 103.7 & 111.4 & 109.9 & 115.5 & 125.5 & 144.2 & 118.0 & 114.7 & 127.6 & 122.3
\end{tabular}

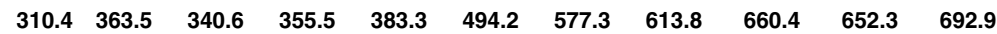

Net international investment position

(UK assets less UK liabilities)

Equity capital

Ordinary share capital and reinvested earnings

Holdings of property

Total equity capital and reinvested earnings

Total other capital

Total

\begin{tabular}{lrrrrrrrrrrr} 
LTNM & 343.5 & 320.0 & 387.1 & 416.3 & 386.5 & 339.1 & 306.0 & 345.8 & 445.1 & 409.5 & 432.3 \\
LTNN & 2.2 & 2.4 & 3.0 & 8.7 & 12.8 & 18.9 & 23.3 & 24.1 & 31.3 & 36.9 & 39.9 \\
\cline { 2 - 10 } HBSH & 345.7 & 322.4 & 390.1 & 425.0 & 399.3 & 358.1 & 329.3 & 369.8 & 476.4 & 446.4 & 472.2 \\
CGKF & -37.3 & -69.0 & -93.5 & -89.4 & -104.6 & -146.4 & -173.5 & -83.9 & -68.0 & -65.0 & -86.6 \\
HBWQ & $\mathbf{3 0 8 . 4}$ & $\mathbf{2 5 3 . 5}$ & $\mathbf{2 9 6 . 6}$ & $\mathbf{3 3 5 . 6}$ & $\mathbf{2 9 4 . 7}$ & $\mathbf{2 1 1 . 7}$ & $\mathbf{1 5 5 . 8}$ & $\mathbf{2 8 5 . 9}$ & $\mathbf{4 0 8 . 4}$ & $\mathbf{3 8 1 . 3}$ & $\mathbf{3 8 5 . 6}$
\end{tabular}

\footnotetext{
1 The latest year's data for foreign direct investment are provisional as these

figures are subject to annual benchmarking.
}

2 Prior to 2002 holdings of quoted share capital were included in series CVVC 


\section{Direct investment ${ }^{1}$}

0.4 Sector analysis

Balance sheets valued at end of year

\begin{tabular}{|c|c|c|c|c|c|c|c|c|c|c|c|c|}
\hline & & 2000 & 2001 & 2002 & 2003 & 2004 & 2005 & 2006 & 2007 & 2008 & 2009 & 2010 \\
\hline \multicolumn{13}{|l|}{$\begin{array}{l}\text { Direct investment abroad } \\
\text { (UK assets) }\end{array}$} \\
\hline \multicolumn{13}{|l|}{ By: } \\
\hline Monetary financial institutions & CVKH & 18.1 & 25.5 & 27.7 & 27.6 & 39.1 & 47.5 & 51.7 & 56.6 & 66.0 & 85.2 & 84.4 \\
\hline Insurance companies & DPYH & 24.3 & 22.8 & 22.0 & 24.9 & 27.1 & 27.7 & 38.8 & 47.1 & 56.1 & 59.9 & 65.9 \\
\hline Other financial intermediaries & CVWH & 34.9 & 37.8 & 42.0 & 44.2 & 32.0 & 32.2 & 35.8 & 68.5 & 72.9 & 75.4 & 82.9 \\
\hline Private non-financial corporations & CVLX & 527.9 & 515.8 & 527.5 & 569.9 & 549.4 & 561.9 & 564.5 & 679.8 & 821.0 & 755.5 & 782.6 \\
\hline Household sector $^{2}$ & $\mathrm{AQHH}$ & 12.0 & 14.3 & 16.5 & 23.2 & 28.7 & 35.7 & 41.9 & 47.3 & 52.5 & 57.6 & 62.7 \\
\hline Total & HBWD & 618.8 & 616.9 & 637.2 & 691.1 & 678.1 & 705.9 & 733.1 & 899.8 & 1068.7 & 1033.6 & 1078.5 \\
\hline
\end{tabular}

Direct investment in the UK

(UK liabilities)

$\ln$

Monetary financial institutions Insurance companies

$\begin{array}{lllllllllll}26.0 & 27.2 & 28.1 & 30.1 & 34.2 & 38.6 & 41.9 & 45.0 & 53.7 & 57.4 & 59.9 \\ 11.7 & 13.0 & 14.1 & 19.4 & 18.8 & 17.5 & 25.0 & 21.6 & 24.1 & 17.4 & 18.1\end{array}$

Other financial intermediaries

Securities dealers

CVSM

11.7

Other

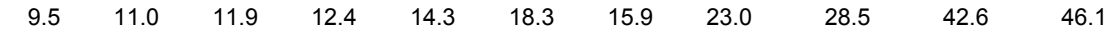

Total other financial intermediaries

CVTC

CVTS

\begin{tabular}{rrrrrrrrrrr}
9.5 & 11.0 & 11.9 & 12.4 & 14.3 & 18.3 & 15.9 & 23.0 & 28.5 & 42.6 & 46.1 \\
15.8 & 27.3 & 29.1 & 30.3 & 31.9 & 26.3 & 46.4 & 57.6 & 36.0 & 39.5 & 40.2 \\
\hline 25.2 & \multirow{2}{*}{38.3} & 41.0 & 42.7 & 46.2 & 44.6 & 62.4 & 80.6 & 64.4 & 82.1 & 86.3
\end{tabular}

Private non-financial corporations

CVUI

$25.2 \quad 38.3$

CVKW

$247.4 \quad 284$

$257.3 \quad 263$

284.1

$310.4 \quad 363.5$

340.6

355.5

383.3

$393.6 \quad 448.0 \quad 466.8$

$518.2 \quad 495.4$

528.7

Total

HBWI

$494.2 \quad 577$

692.9

Net international investment position (UK assets less UK liabilities)

Monetary financial institutions Insurance companies

LTNP

Other financial intermediares

Private non-financial corporations

Public corporations

LTNP

LTNQ

CVOF

Household sector ${ }^{2}$

$\mathrm{AQHH}$

$\begin{array}{rr}-8.0 & -1.7 \\ 12.6 & 9.8 \\ 9.7 & -0.5 \\ 280.4 & 230.8 \\ 1.7 & 0.8\end{array}$

$-0.5-2.5$

$\begin{array}{rr}1.7 & 0.8 \\ 12.0 & 14.3\end{array}$

1.0
270.2

$\begin{array}{rrrrr}5.4 & 4.9 & 8.9 & 9.9 & 11.6 \\ 1.4 & 8.3 & 10.2 & 13.7 & 25.6\end{array}$

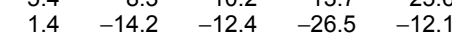

$\begin{array}{rrrrrr}1.5 & 1.4 & 1.7 & 0.8 & 0.3 & 0.4 \\ 16.5 & 23.2 & 28.7 & 35.7 & 41.9 & 47.3\end{array}$

$\begin{array}{llllllll}308.4 & 253.5 & 296.6 & 335.6 & 294.7 & 211.7 & 155.8 & 285.9\end{array}$

$\begin{array}{rrr}12.3 & 27.8 & 24.5 \\ 32.1 & 42.5 & 47.7 \\ 8.4 & -6.6 & -3.4 \\ 302.8 & 260.0 & 253.9 \\ 0.2 & - & 0.1 \\ 52.5 & 57.6 & 62.7 \\ & & \\ \mathbf{4 0 8 . 4} & \mathbf{3 8 1 . 3} & \mathbf{3 8 5 . 6}\end{array}$

1 The latest year's data for foreign direct investment are provisional as these

figures are subject to annual benchmarking.

2 The household sector includes non-profit institutions serving households. 


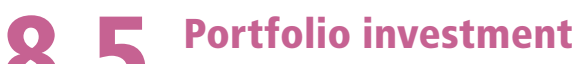

\section{(O) Balance sheets valued at end of year}

\begin{tabular}{|c|c|c|c|c|c|c|c|c|c|c|c|c|}
\hline & & & & & & & & & & & & billion \\
\hline & & 2000 & 2001 & 2002 & 2003 & 2004 & 2005 & 2006 & 2007 & 2008 & 2009 & 2010 \\
\hline $\begin{array}{l}\text { Portfolio investment abroad } \\
\text { (UK assets) }\end{array}$ & & & & & & & & & & & & \\
\hline Investment in equity securities (shares) by: & & & & & & & & & & & & \\
\hline Monetary financial institutions & VTWF & 19.7 & 14.3 & 2.7 & 20.8 & 53.0 & 86.2 & 109.2 & 127.0 & 41.7 & 49.7 & 63.5 \\
\hline Insurance companies and pension funds & & & & & & & & & & & & \\
\hline Insurance companies ${ }^{1}$ & CGPB & 100.7 & 106.2 & 82.1 & 79.1 & 91.4 & 132.0 & 150.2 & 178.6 & 149.9 & 169.0 & 188.1 \\
\hline Self-administered pension funds & ZPOR & 135.5 & 127.9 & 104.4 & 125.7 & 140.3 & 192.1 & 202.0 & 178.6 & 136.5 & 164.6 & 181.4 \\
\hline Total insurance companies and pension funds & CGPV & 236.2 & 234.1 & 186.5 & 204.9 & 231.7 & 324.0 & 352.1 & 357.2 & 286.4 & 333.5 & 369.6 \\
\hline Other financial intermediaries & & & & & & & & & & & & \\
\hline Securities dealers & HCEA & 49.3 & 46.8 & 22.9 & 32.4 & 46.3 & 33.9 & 31.3 & 50.2 & 60.7 & 62.9 & 63.3 \\
\hline Other & CGTV & - & - & - & - & - & - & - & 1.0 & 1.0 & 1.0 & 1.0 \\
\hline Total other financial intermediaries & HDIG & 148.4 & 134.8 & 100.2 & 126.5 & 146.2 & 164.9 & 182.9 & 214.1 & 196.3 & 241.2 & 270.1 \\
\hline Private non-financial corporations & XBNN & 10.0 & 8.9 & 6.5 & 7.8 & 7.9 & 9.3 & 11.6 & 11.3 & 9.1 & 10.3 & 11.3 \\
\hline Household sector $^{2}$ & HFLX & 15.0 & 12.5 & 10.0 & 12.0 & 16.5 & 32.6 & 37.3 & 39.1 & 31.5 & 31.5 & 29.1 \\
\hline Total investment in equity securities & HEPX & 429.3 & 404.6 & 305.9 & 372.0 & 455.3 & 617.2 & 693.4 & 748.9 & 565.2 & 666.4 & 743.8 \\
\hline
\end{tabular}

Investment in debt securities

Investment in bonds and notes by:

Monetary financial institutions

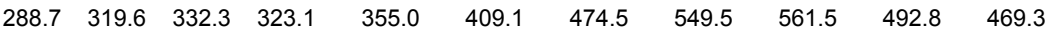

Central Government

HQ50

Insurance companies and pension funds

Insurance companies

Self-administered pension funds

CGTU

Total insurance companies and pension funds HBUM

Other financial intermediaries

Securities dealers

Unit and investment trusts

Other

Total other financial intermediaries

Private non-financial corporations

Household sector ${ }^{2}$

Total investment in bonds and notes

HCDZ

HBXZ

HCNA

HCOR

XBNK

HCJC

HEPW

\begin{tabular}{rrrrrrrrrrr}
39.8 & 55.9 & 62.9 & 64.5 & 77.0 & 80.5 & 103.6 & 114.7 & 141.4 & 165.6 & 170.9 \\
44.2 & 49.9 & 45.7 & 53.9 & 64.6 & 87.0 & 107.9 & 141.7 & 143.2 & 164.2 & 209.0 \\
\hline 84.0 & 105.8 & 108.6 & 118.3 & 141.7 & 167.6 & 211.4 & 256.4 & 284.6 & 329.8 & 379.9 \\
& & & & & & & & & & \\
45.2 & 34.9 & 31.1 & 38.7 & 57.3 & 73.8 & 40.6 & 17.9 & 133.5 & 239.7 & 320.2 \\
6.8 & 8.4 & 7.7 & 10.7 & 13.4 & 20.6 & 29.8 & 34.1 & 37.2 & 50.3 & 60.8 \\
- & - & - & - & - & - & - & - & - & - & - \\
\hline 52.1 & 43.3 & 38.8 & 49.4 & 70.7 & 94.4 & 70.4 & 52.0 & 170.7 & 290.0 & 381.0 \\
1.6 & 2.0 & 2.2 & 3.5 & 3.7 & 3.0 & 1.3 & 2.0 & 4.7 & 3.4 & 6.5 \\
7.5 & 7.6 & 7.8 & 7.7 & 7.7 & 7.7 & 7.5 & 7.6 & 8.5 & 8.2 & 8.3 \\
\hline 433.8 & 478.3 & 489.6 & 502.0 & 578.8 & 681.7 & 765.2 & 867.5 & 1030.0 & 1124.4 & 1245.2
\end{tabular}

Investment in Money Market Instruments by:

Central government

Monetary financial institutions

Insurance companies and pension funds

Other financial intermediaries

Private non-financial corporations

Total investment in Money Market Instruments

Total investment in debt securities

\begin{tabular}{|c|c|c|c|c|c|c|c|c|c|c|c|}
\hline LSPI & - & 0.5 & 0.9 & - & - & - & - & 2.4 & 2.3 & 4.9 & 5.5 \\
\hline HHZV & 34.9 & 42.1 & 34.6 & 42.8 & 37.0 & 39.6 & 42.8 & 53.8 & 57.1 & 65.4 & 65.0 \\
\hline HBXX & 1.3 & 1.1 & 1.4 & 1.5 & 2.1 & 3.5 & 3.0 & 3.1 & 3.8 & 4.0 & 3.9 \\
\hline JXG4 & 3.8 & 6.0 & 5.6 & 7.8 & 8.5 & 7.5 & 10.5 & 7.6 & 4.6 & 7.3 & 7.8 \\
\hline HFBN & 3.0 & 4.9 & 6.0 & 9.8 & 10.4 & 11.4 & 16.2 & 6.4 & 1.3 & 2.9 & 4.9 \\
\hline HLYR & 43.0 & 54.5 & 48.5 & 61.9 & 58.0 & 62.0 & 72.5 & 73.3 & 69.1 & 84.5 & 87.1 \\
\hline HHZX & 476.8 & 532.8 & 538.1 & 563.9 & 636.8 & 743.8 & 837.7 & 940.8 & 1099.1 & 1208.9 & 1332.3 \\
\hline HHZZ & 906.1 & 937.4 & 844.0 & 935.8 & 1092.1 & 1360.9 & 1531.0 & 1689.7 & 1664.3 & 1875.3 & 2076.1 \\
\hline
\end{tabular}

1 Includes pension funds managed by insurance companies.

2 The household sector includes non-profit institutions serving households. 


\section{( 5 Portfolio investment}

continued

Balance sheets valued at end of year

\section{Portfolio investment in the UK \\ (UK liabilities) \\ Investment in equity securities (shares) issued by: \\ Monetary financial institutions}

Other sectors ${ }^{1}$

Total investment in equity securities

\begin{tabular}{|c|c|c|c|c|c|c|c|c|c|c|c|}
\hline $\begin{array}{l}\text { BQD } \\
3 Q E\end{array}$ & $\begin{array}{r}6.6 \\
667.1\end{array}$ & $\begin{array}{r}5.6 \\
578.5\end{array}$ & $\begin{array}{r}3.2 \\
439.5\end{array}$ & $\begin{array}{r}4.5 \\
522.6\end{array}$ & $\begin{array}{r}4.6 \\
570.1\end{array}$ & $\begin{array}{r}5.3 \\
654.0\end{array}$ & $\begin{array}{r}7.0 \\
773.8\end{array}$ & $\begin{array}{r}11.0 \\
824.8\end{array}$ & $\begin{array}{r}8.8 \\
596.7\end{array}$ & $\begin{array}{r}14.2 \\
839.4\end{array}$ & $\begin{array}{r}17.7 \\
926.8\end{array}$ \\
\hline & 673.7 & 584.1 & 442.7 & 527.1 & 574.7 & 659.3 & 780.8 & 835.8 & 605.5 & 853.6 & 144 \\
\hline
\end{tabular}

Investment in debt securities

Investment in bonds and notes

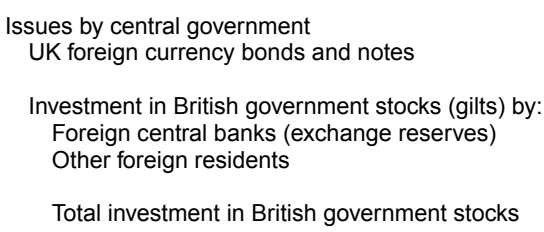

Total monetary financial institutions

Issues by other sectors

Total investment in bonds and notes

Investment in Money Market Instruments

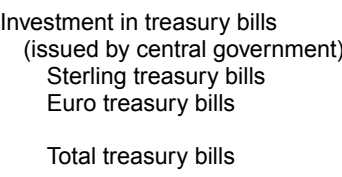

Investment in certificates of deposit issued by UK monetary financial institutions

Investment in commercial paper Issued by UK monetary financial Institutions Issued by other sectors ${ }^{1}$

Total investment in commercial paper

Total investment in Money Market Instruments

Total investment in debt securities

\begin{tabular}{lrrrrrrrrrrrr} 
HEWE & 6.5 & 3.3 & 0.9 & 1.6 & 1.5 & 1.7 & 1.5 & 1.5 & - & - & - \\
& & & & & & & & & & & \\
HCCH & 18.1 & 18.7 & 17.3 & 15.9 & 21.0 & 21.0 & 25.2 & 29.2 & 36.4 & 49.1 & 61.2 \\
HEQF & 37.8 & 37.8 & 38.2 & 48.6 & 61.3 & 88.0 & 108.8 & 129.5 & 167.3 & 175.1 & 248.0 \\
\cline { 2 - 10 } HEWD & 55.9 & 56.5 & 55.5 & 64.5 & 82.3 & 109.0 & 134.0 & 158.7 & 203.6 & 224.3 & 309.2 \\
HHGF & 62.4 & 59.9 & 56.4 & 66.1 & 83.8 & 110.7 & 135.5 & 160.2 & 203.6 & 224.3 & 309.2 \\
HHGG & - & - & - & - & - & - & - & - & - & - & - \\
HEWM & - & - & - & - & - & - & - & - & - & - & - \\
& & & & & & & & & & & \\
HMBL & 39.0 & 41.6 & 51.4 & 68.0 & 83.0 & 105.9 & 119.3 & 139.2 & 158.2 & 180.8 & 179.7 \\
& & & & & & & & & & & \\
HHGI & 38.4 & 42.5 & 43.6 & 53.7 & 70.9 & 95.5 & 114.9 & 166.9 & 200.2 & 208.0 & 204.0 \\
HMBF & 77.4 & 84.1 & 95.0 & 121.7 & 154.0 & 201.3 & 234.1 & 306.2 & 358.4 & 388.8 & 383.7 \\
HHGJ & 121.3 & 129.5 & 160.1 & 211.7 & 258.3 & 329.3 & 371.3 & 433.4 & 559.4 & 630.0 & 626.4 \\
HLXZ & 261.1 & 273.4 & 311.5 & 399.5 & 496.1 & 641.3 & 740.8 & 899.8 & 1121.4 & 1243.1 & 1319.2
\end{tabular}

ACOJ HHNX

HLYU

\begin{tabular}{rrrrrrrrrrr}
- & 0.1 & 0.2 & 1.9 & 3.8 & 2.8 & 3.5 & 7.2 & 21.0 & 19.4 & 27.9 \\
- & - & - & - & - & - & - & - & - & - & - \\
\hline- & 0.1 & 0.2 & 1.9 & 3.8 & 2.8 & 3.5 & 7.2 & 21.0 & 19.4 & 27.9
\end{tabular}

HHGM

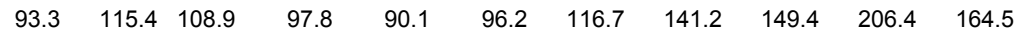

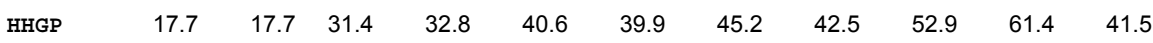

HLYQ

$\begin{array}{lllllllllll}21.7 & 22.5 & 30.6 & 23.7 & 22.5 & 22.2 & 16.3 & 19.1 & 29.9 & 21.5 & 25.4 \\ 39.4 & 40.2 & 62.0 & 56.5 & 63.2 & 62.1 & 61.5 & 61.6 & 82.8 & 82.9 & 66.8\end{array}$

$H H G R$

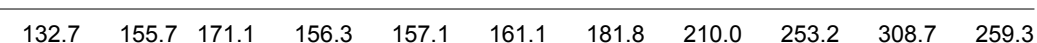

HLXY

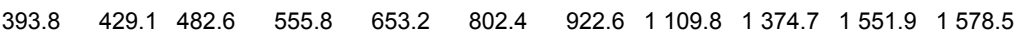

HLXW

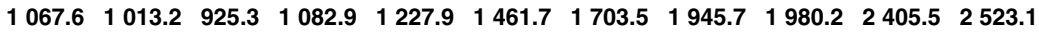

1 These series relate to non-governmental sectors other than monetary financial institutions. 


\title{
85 Portfolio investment
}

continued

Balance sheets valued at end of year

\begin{tabular}{|c|c|c|c|c|c|c|c|c|c|c|c|c|}
\hline & & 2000 & 2001 & 2002 & 2003 & 2004 & 2005 & 2006 & 2007 & 2008 & 2009 & 2010 \\
\hline \multicolumn{13}{|c|}{$\begin{array}{l}\text { Net international investment position } \\
\text { (UK assets less UK liabilities) }\end{array}$} \\
\hline Equity securities & CGNE & -244.4 & -179.4 & -136.8 & -155.1 & -119.4 & -42.1 & -87.5 & -86.9 & -40.3 & -187.2 & -200.8 \\
\hline \multicolumn{13}{|l|}{ Debt securities } \\
\hline Bonds and notes & LTNS & 172.7 & 204.9 & 178.1 & 102.5 & 82.7 & 40.4 & $\begin{array}{r}24.4 \\
-1093\end{array}$ & $\begin{array}{r}-32.3 \\
-1367\end{array}$ & $\begin{array}{r}-91.4 \\
-1841\end{array}$ & $\begin{array}{l}-118.8 \\
-2242\end{array}$ & $\begin{array}{r}-74.0 \\
-172 \\
1\end{array}$ \\
\hline Money market instruments & LTNT & -89.8 & -101.2 & -122.6 & -94.4 & -99.1 & -99.1 & -109.3 & -136.7 & -184.1 & -224.2 & -172.1 \\
\hline Total & CGNH & -161.5 & -75.7 & -81.3 & -147.0 & -135.8 & -100.8 & -172.4 & -255.9 & -315.9 & -530.2 & -447.0 \\
\hline
\end{tabular}

\section{Portfolio investment \\ 8.6 Sector analysis}

\section{Balance sheets valued at end of year}

\begin{tabular}{|c|c|c|c|c|c|c|c|c|c|c|c|c|}
\hline & & 2000 & 2001 & 2002 & 2003 & 2004 & 2005 & 2006 & 2007 & 2008 & 2009 & 2010 \\
\hline \multicolumn{13}{|l|}{$\begin{array}{l}\text { Portfolio investment abroad } \\
\text { (UK assets) }\end{array}$} \\
\hline \multicolumn{13}{|l|}{ Investment by: } \\
\hline Monetary financial institutions & HHGQ & 343.2 & 376.0 & 369.7 & 386.7 & 445.0 & 534.8 & 626.5 & 730.4 & 660.4 & 607.9 & 597.8 \\
\hline Central government & LOFC & - & 0.5 & 0.9 & - & - & 0.2 & 0.2 & 2.6 & 2.5 & 5.3 & 5.8 \\
\hline Insurance companies and pension funds & HHHH & 321.4 & 341.0 & 296.5 & 324.7 & 375.4 & 495.1 & 566.6 & 616.7 & 574.8 & 667.4 & 753.4 \\
\hline Other financial intermediaries & HHNH & 204.3 & 184.0 & 144.5 & 183.7 & 225.5 & 266.8 & 263.8 & 273.6 & 371.6 & 538.4 & 658.9 \\
\hline Private non-financial corporations & AIMH & 14.7 & 15.8 & 14.6 & 21.0 & 21.9 & 23.7 & 29.2 & 19.7 & 15.1 & 16.7 & 22.8 \\
\hline Household sector $^{1}$ & AINA & 22.4 & 20.2 & 17.7 & 19.7 & 24.2 & 40.3 & 44.8 & 46.7 & 40.0 & 39.6 & 37.4 \\
\hline Total & HHzZ & 906.1 & 937.4 & 844.0 & 935.8 & 1092.1 & 1360.9 & 1531.0 & 1689.7 & 1664.3 & 1875.3 & 2076.1 \\
\hline
\end{tabular}

Portfolio investment in the UK

(UK liabilities)

Investment in securities issued by:

Monetary financial institutions

Central government

Local authorities

Public corporations

Other sectors

Total

\begin{tabular}{|c|c|c|c|c|c|c|c|c|c|c|c|}
\hline CGPC & 195.0 & 222.8 & 238.6 & 256.8 & 289.3 & 342.7 & 403.1 & 500.9 & 569.5 & 670.9 & 607.4 \\
\hline HHGS & 62.4 & 60.0 & 56.5 & 68.0 & 87.6 & 113.4 & 139.0 & 167.4 & 224.6 & 243.7 & 337.1 \\
\hline HHGG & - & - & - & - & - & - & - & - & - & - & - \\
\hline HEWM & - & - & - & - & - & - & - & - & - & - & - \\
\hline CGPG & 810.2 & 730.5 & 630.2 & 758.0 & 851.0 & 1005.5 & 1161.4 & 1277.4 & 1186.0 & 1490.9 & 1578.6 \\
\hline HLXW & 1067.6 & 1013.2 & 925.3 & 1082.9 & 1227.9 & 1461.7 & 1703.5 & 1945.7 & 1980.2 & 2405.5 & 2523.1 \\
\hline
\end{tabular}

Net international investment position

(UK assets less UK liabilities)

\author{
Monetary financial institutions \\ Central government \\ Local authorities \\ Public corporations \\ Other sectors \\ Total
}

$\begin{array}{rrrrrrrrrrrr}\text { LTNU } & 148.3 & 153.2 & 131.1 & 129.8 & 155.7 & 192.1 & 223.4 & 229.5 & 90.8 & -63.0 & -9.6 \\ \text { ZPOH } & -62.4 & -59.5 & -55.6 & -68.0 & -87.6 & -113.3 & -138.8 & -164.7 & -222.2 & -238.4 & -331.3 \\ \text { HHGG } & - & - & - & - & - & - & - & - & - & - & - \\ \text {-HEWM } & - & - & - & - & - & - & - & - & - & - & - \\ \text { LTNV } & -247.3 & -169.5 & -156.7 & -208.8 & -203.9 & -179.6 & -257.1 & -320.7 & -184.6 & -228.7 & -106.1 \\ & & & & & & & & & & -\mathbf{1 0} & \\ \text { CGNH } & -\mathbf{1 6 1 . 5} & -\mathbf{7 5 . 7} & -\mathbf{8 1 . 3} & -\mathbf{1 4 7 . 0} & -\mathbf{1 3 5 . 8} & \mathbf{- 1 0 0 . 8} & -\mathbf{1 7 2 . 4} & \mathbf{- 2 5 5 . 9} & \mathbf{- 3 1 5 . 9} & \mathbf{- 5 3 0 . 2} & -\mathbf{4 4 7 . 0}\end{array}$

1 The household sector includes non-profit institutions serving households. 


\subsection{Ontrerinestrent}

Balance sheets valued at end of year

\begin{tabular}{rlllllllllll}
\hline & 2000 & 2001 & 2002 & 2003 & 2004 & 2005 & 2006 & 2007 & 2008 & 2009 & 2010
\end{tabular}

Other investment abroad

(UK assets)

Trade credit

\section{Long-term \\ Central government \\ Other sectors $^{1}$ \\ Total long-term trade credit}

Short-term

Other sectors ${ }^{1}$

Total trade credit

Loans

Long-term

Bank loans under ECGD ${ }^{2}$ guarantee Inter-government loans by the UK and

other central government assets

Loans by Commonwealth Development

Corporation (public corporations)

Loans by the ECGD ${ }^{2}$

Loans by specialist leasing companies ${ }^{1}$

Total long-term loans

Short-term

By monetary financial institutions

Sterling loans

Foreign currency loans

Total monetary financial institutions

By other sectors

Total short-term loans

Total loans

$\begin{array}{lrrrrrrrrrrr}\text { HCFQ } & 4.8 & 5.1 & 3.8 & 3.7 & 3.7 & 4.1 & 3.3 & 3.2 & 4.4 & 4.1 & 4.5 \\ \text { HCFN } & 0.2 & 0.2 & 0.2 & 0.2 & 0.2 & 0.2 & 0.1 & 0.1 & 0.1 & - & - \\ & & & & & & & & & & & \\ \text { HEWZ } & 0.5 & 0.4 & 0.4 & 0.4 & 0.3 & 0.3 & 0.3 & 0.3 & 0.3 & 0.3 & 0.3 \\ \text { CY94 } & 2.4 & 2.6 & 2.4 & 2.4 & 2.6 & 2.2 & 1.1 & 1.0 & 1.0 & 0.9 & 0.8 \\ \text { HGIH } & - & - & - & - & - & - & - & - & - & - & - \\ \text { HFAX } & 8.0 & 8.3 & 6.8 & 6.6 & 6.8 & 6.8 & 4.9 & 4.6 & 5.8 & 5.3 & 5.6\end{array}$

\begin{tabular}{lrrrrrrrrrrr} 
JXG7 & 27.5 & 32.2 & 37.4 & 40.2 & 47.4 & 66.9 & 87.7 & 113.1 & 117.8 & 96.6 & 103.4 \\
JXG8 & 252.4 & 290.9 & 290.9 & 358.3 & 448.9 & 575.1 & 621.3 & 842.8 & 1010.0 & 806.9 & 867.9 \\
\cline { 2 - 10 } ZPOM & 279.9 & 323.0 & 328.4 & 398.4 & 496.3 & 642.0 & 709.1 & 955.9 & 1127.8 & 903.5 & 971.2 \\
HLXI & 0.5 & 0.5 & 0.5 & 0.5 & 0.5 & 0.5 & 0.5 & 0.5 & 1.3 & 0.5 & 0.5 \\
\cline { 2 - 10 } VTUM & 280.5 & 323.6 & 328.9 & 399.0 & 496.9 & 642.6 & 709.6 & 956.4 & 1129.1 & 904.0 & 971.8 \\
& & & & & & & & & & & \\
HLXQ & 288.5 & 331.9 & 335.7 & 405.6 & 503.7 & 649.4 & 714.5 & 961.1 & 1134.8 & 909.4 & 977.4
\end{tabular}

Currency and deposits

Foreign notes and coin

Monetary financial institutions

Other sectors ${ }^{1}$

Total foreign notes and coin

Deposits abroad by UK residents

Deposits by monetary financial institutions

Sterling deposits

Foreign currency deposits

Total monetary financial institutions

Deposits by securities dealers

Deposits by other UK residents ${ }^{1}$

Total deposits abroad

TAAF

CGML

HEOX

\begin{tabular}{lllllllllll}
0.1 & 0.1 & 0.1 & 0.1 & 0.1 & 0.1 & 0.2 & 0.2 & 0.4 & 0.3 & 0.2 \\
0.4 & 0.4 & 0.4 & 0.5 & 0.5 & 0.5 & 0.5 & 0.6 & 0.6 & 0.5 & 0.5 \\
\hline 0.5 & 0.5 & 0.5 & 0.6 & 0.6 & 0.6 & 0.7 & 0.8 & 0.9 & 0.8 & 0.7
\end{tabular}

$\begin{array}{llllllllllll}\text { JXG9 } & 97.4 & 104.7 & 96.6 & 114.9 & 112.0 & 146.1 & 193.3 & 304.8 & 279.1 & 210.4 & 185.1\end{array}$ JXH2

VTWL

HGUX HHGW

HBXS

\begin{tabular}{rrrrrrrrrrr}
97.4 & 104.7 & 96.6 & 114.9 & 112.0 & 146.1 & 193.3 & 304.8 & 279.1 & 210.4 & 185.1 \\
678.2 & 700.6 & 746.5 & 806.6 & 894.9 & 1053.5 & 1092.6 & 1409.4 & 1813.5 & 1568.9 & 1691.2 \\
\hline 775.6 & 805.3 & 843.0 & 921.4 & 1006.9 & 1199.7 & 1286.0 & 1714.1 & 2092.6 & 1779.3 & 1876.3 \\
& & & & & & & & & & \\
206.1 & 261.9 & 242.0 & 289.7 & 315.7 & 497.4 & 504.8 & 555.7 & 354.8 & 331.3 & 387.5 \\
98.9 & 112.3 & 112.4 & 183.9 & 279.3 & 356.5 & 397.6 & 497.1 & 577.5 & 474.2 & 528.3 \\
\hline 1080.6 & 1179.5 & 1197.4 & 1395.0 & 1602.0 & 2053.5 & 2188.3 & 2767.0 & 3024.9 & 2584.7 & 2792.1
\end{tabular}

HBVS

1 These series relate to non-governmental sectors other than monetary financial

institutions.

2 Export Credits Guarantee Department 


\section{Other investment}

Balance sheets valued at end of year

\begin{tabular}{|c|c|c|c|c|c|c|c|c|c|c|c|c|}
\hline \multicolumn{2}{|l|}{ continued } & \multirow[b]{2}{*}{2000} & \multirow[b]{2}{*}{2001} & \multirow[b]{2}{*}{2002} & \multirow[b]{2}{*}{2003} & \multirow[b]{2}{*}{2004} & \multirow[b]{2}{*}{2005} & \multirow[b]{2}{*}{2006} & \multirow[b]{2}{*}{2007} & \multirow[b]{2}{*}{2008} & \multicolumn{2}{|r|}{$£$ billion } \\
\hline & & & & & & & & & & & 2009 & 2010 \\
\hline $\begin{array}{l}\text { Other investment abroad - continued } \\
\text { (UK assets) }\end{array}$ & & & & & & & & & & & & \\
\hline $\begin{array}{l}\text { Other assets } \\
\text { Central government assets } \\
\text { Central government subscriptions to } \\
\text { international organisations }\end{array}$ & & & & & & & & & & & & \\
\hline Regional development banks & HEXW & 1.1 & 1.1 & 1.2 & 1.3 & 1.3 & 1.4 & 1.5 & 1.5 & 1.7 & 1.9 & 2.0 \\
\hline European Investment Bank (EIB) & HEXX & 0.4 & 0.4 & 0.4 & 0.4 & 0.4 & 0.4 & 0.4 & 0.4 & 0.4 & 0.4 & 0.4 \\
\hline Other subscriptions & HEXZ & 0.4 & 0.4 & 0.4 & 0.4 & 0.5 & 0.5 & 0.7 & 0.9 & 0.9 & 1.0 & 1.0 \\
\hline Total central government subscriptions & HLXO & 6.8 & 7.1 & 7.5 & 7.7 & 8.0 & 8.5 & 9.2 & 10.0 & 11.0 & 12.0 & 13.3 \\
\hline Other long-term central government assets & XBJL & - & - & - & - & - & - & - & - & - & - & - \\
\hline Other short-term central government assets & LOEM & 1.8 & 1.7 & 2.3 & 2.5 & 1.9 & 2.4 & 2.6 & 3.0 & 5.6 & 6.1 & 6.3 \\
\hline Total central government & LOET & 8.6 & 8.8 & 9.8 & 10.2 & 10.0 & 10.9 & 11.9 & 13.1 & 16.6 & 18.2 & 19.6 \\
\hline \multicolumn{13}{|l|}{ Other sectors assets } \\
\hline Long-term assets ${ }^{1}$ & HLXM & - & - & - & - & - & - & - & - & - & - & - \\
\hline \multicolumn{13}{|l|}{ Short-term assets } \\
\hline Public corporations assets abroad & HGJM & - & - & - & - & - & - & - & - & - & - & - \\
\hline Other $^{1}$ & HHGY & 1.1 & 1.1 & 1.4 & 1.3 & 1.1 & 1.1 & 0.6 & 1.9 & 2.5 & 3.3 & 3.9 \\
\hline Total short-term assets & HLXJ & 1.1 & 1.1 & 1.4 & 1.3 & 1.1 & 1.1 & 0.6 & 1.9 & 2.5 & 3.3 & 3.9 \\
\hline Total other sectors & HLXN & 1.1 & 1.1 & 1.4 & 1.3 & 1.1 & 1.1 & 0.6 & 1.9 & 2.5 & 3.3 & 3.9 \\
\hline Total other assets & HLXS & 9.7 & 10.0 & 11.2 & 11.5 & 11.0 & 12.0 & 12.5 & 15.0 & 19.1 & 21.4 & 23.5 \\
\hline Total & HLXV & 1379.7 & 1521.9 & 1545.2 & 1813.7 & 2118.0 & 2714.8 & 2916.6 & 3744.5 & 4180.6 & 3517.1 & 3794.3 \\
\hline
\end{tabular}




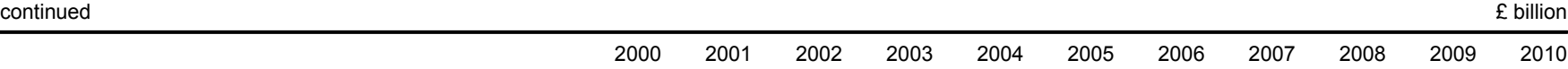

Other investment in the UK

HBWC

Trade credit

Short-term

Total trade credit

Loans

Long-term loans to:

Central government

Local authorities

Public corporations

Other $^{1}$

Total long-term loans

Short-term loans to:

Central government

Local authorities

Securities dealers

Other $^{1}$

Total short-term loans

Total loans

Currency and deposits

Sterling notes and coin

Notes (issued by Bank of England)

Coins (issued by central government)

Total notes and coin

Deposits from abroad with UK residents

Deposits with monetary financial institutions

Sterling deposits

Foreign currency deposits

Total deposits with UK monetary

financial institutions

Deposit liabilities of UK central government

Total deposits from abroad with UK residents HBYA

Total currency and deposits

HLVI

HLVG

HLVH

APME

\begin{tabular}{lllllllllll}
1.0 & 1.0 & 1.1 & 1.1 & 1.2 & 1.3 & 1.4 & 1.4 & 1.4 & 1.2 & 1.3 \\
0.1 & 0.1 & 0.1 & 0.1 & 0.1 & 0.1 & 0.2 & 0.2 & 0.2 & 0.1 & 0.1 \\
\hline 1.1 & 1.1 & 1.2 & 1.3 & 1.4 & 1.4 & 1.5 & 1.5 & 1.5 & 1.4 & 1.4
\end{tabular}

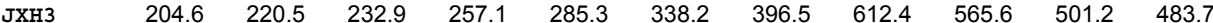
JXH4

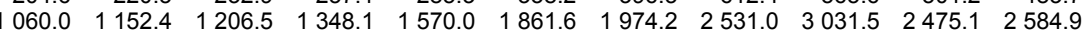

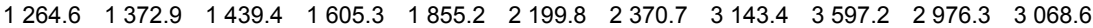

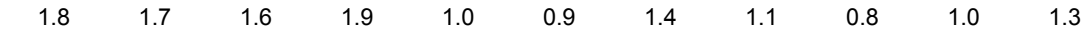

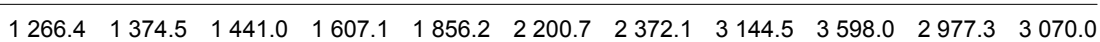

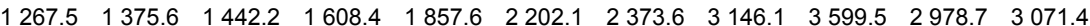

Other liabilities

Long-term

Net equity of foreign households in life

assurance reserves and pension funds

Prepayments of insurance premiums and

reserves for oustanding claims

Total long-term liabilities

$\begin{array}{llllllllll}0.2 & 0.2 & 0.2 & 0.2 & 0.2 & 0.2 & 0.2 & 0.2 & 0.2 & 0.2\end{array}$

NQLR

VTUF

HBMV

Short-term

Total other liabilities

HLYM

HLYD

\begin{tabular}{rrrrrrrrrrr}
10.8 & 10.7 & 12.6 & 10.2 & 11.5 & 14.2 & 18.6 & 14.4 & 10.5 & 10.2 & 10.4 \\
\hline 11.0 & 10.9 & 12.9 & 10.4 & 11.7 & 14.4 & 18.8 & 14.6 & 10.7 & 10.4 & 10.6 \\
1.1 & 1.6 & 1.0 & 1.0 & 0.9 & 1.0 & 1.1 & 1.3 & 1.5 & 1.6 & 2.4 \\
\hline 12.0 & 12.5 & 13.8 & 11.4 & 12.6 & 15.5 & 19.9 & 16.0 & 12.2 & 12.0 & 13.0
\end{tabular}

1 These series relate to non-governmental sectors other than monetary financial institutions. 


\section{Other investment}

continued

Balance sheets valued at end of year

\begin{tabular}{|c|c|c|c|c|c|c|c|c|c|c|c|c|}
\hline \multirow[t]{2}{*}{ continued } & & & & & & & & & & & & $£$ billion \\
\hline & & 2000 & 2001 & 2002 & 2003 & 2004 & 2005 & 2006 & 2007 & 2008 & 2009 & 2010 \\
\hline \multicolumn{13}{|c|}{$\begin{array}{l}\text { Net international investment position } \\
\text { (UK assets less UK liabilities) }\end{array}$} \\
\hline Trade credit & LTNW & -0.7 & -1.0 & -0.6 & 0.1 & -0.2 & -1.7 & -0.2 & -0.1 & -0.4 & -0.3 & -0.4 \\
\hline Loans & LTNX & -82.5 & -140.7 & -105.3 & -108.8 & -137.1 & -241.1 & -161.1 & 8.4 & 229.6 & 109.1 & 15.2 \\
\hline Currency and deposits & LTNY & -186.5 & -195.7 & -244.2 & -212.8 & -254.9 & -148.0 & -184.6 & -378.3 & -573.7 & -393.2 & -278.7 \\
\hline Other & LTNZ & -2.3 & -2.5 & -2.7 & 0.1 & -1.6 & -3.5 & -7.4 & -1.0 & 6.9 & 9.4 & 10.5 \\
\hline
\end{tabular}

\section{Other investment}

8.8 Sector analysis

Balance sheets valued at end of year

\begin{tabular}{|c|c|c|c|c|c|c|c|c|c|c|c|c|}
\hline & & 2000 & 2001 & 2002 & 2003 & 2004 & 2005 & 2006 & 2007 & 2008 & 2009 & 2010 \\
\hline \multicolumn{13}{|l|}{$\begin{array}{l}\text { Other investment abroad } \\
\text { (UK assets) }\end{array}$} \\
\hline \multicolumn{13}{|l|}{ Investment by: } \\
\hline Monetary financial institutions & VTXD & 1060.5 & 1133.5 & 1175.3 & 1323.6 & 1507.1 & 1845.9 & 1998.5 & 2673.4 & 3225.2 & 2687.2 & 2852.2 \\
\hline Central government & CGEN & 8.9 & 9.1 & 10.0 & 10.4 & 10.1 & 11.0 & 12.1 & 13.2 & 16.7 & 18.2 & 19.7 \\
\hline Public corporations & CGEO & 2.9 & 3.0 & 2.8 & 2.8 & 2.9 & 2.5 & 1.4 & 1.3 & 1.3 & 1.2 & 1.0 \\
\hline Other sectors & $\mathrm{CGGH}$ & 307.4 & 376.3 & 357.1 & 476.8 & 597.8 & 855.3 & 904.6 & 1056.6 & 937.4 & 810.5 & 921.4 \\
\hline Total & HLXV & 1379.7 & 1521.9 & 1545.2 & 1813.7 & 2118.0 & 2714.8 & 2916.6 & 3744.5 & 4180.6 & 3517.1 & 3794.3 \\
\hline \multicolumn{13}{|l|}{$\begin{array}{l}\text { Other investment in the UK } \\
\text { (UK liabilities) }\end{array}$} \\
\hline \multicolumn{13}{|l|}{ Investment in: } \\
\hline Monetary financial institutions & CGHB & 1265.6 & 1373.9 & 1440.5 & 1606.4 & 1856.4 & 2201.0 & 2372.0 & 3144.8 & 3598.6 & 2977.5 & 3069.9 \\
\hline Central government & CGHG & 3.2 & 3.4 & 2.6 & 2.8 & 1.7 & 1.8 & 2.3 & 2.2 & 2.1 & 2.4 & 3.5 \\
\hline Local authorities & CGHX & 0.8 & 0.8 & 0.9 & 1.1 & 1.7 & 1.9 & 2.1 & 2.1 & 2.5 & 3.0 & 3.2 \\
\hline Other sectors & CGNC & 382.1 & 483.8 & 454.1 & 524.8 & 651.5 & 903.9 & 893.2 & 966.0 & 914.5 & 808.7 & 970.6 \\
\hline Total & HLYD & 1651.6 & 1861.9 & 1898.0 & 2135.2 & 2511.8 & 3109.0 & 3270.0 & 4115.6 & 4518.1 & 3792.0 & 4047.6 \\
\hline \multicolumn{13}{|c|}{$\begin{array}{l}\text { Net international investment position } \\
\text { (UK assets less UK liabilities) }\end{array}$} \\
\hline Monetary financial institutions & LтоC & -205.1 & -240.3 & -265.1 & -282.8 & -349.4 & -355.1 & -373.6 & -471.4 & -373.4 & -290.3 & -217.7 \\
\hline Central government & LTOD & 5.7 & 5.7 & 7.4 & 7.6 & 8.4 & 9.3 & 9.8 & 11.0 & 14.6 & 15.8 & 16.1 \\
\hline Local authorities & - CGHX & -0.8 & -0.8 & -0.9 & -1.1 & -1.7 & -1.9 & -2.1 & -2.1 & -2.5 & -3.0 & -3.2 \\
\hline Public corporations & LTOE & 2.9 & 3.0 & 2.8 & 2.8 & 2.5 & 2.1 & 1.0 & 0.9 & 0.9 & 0.8 & 0.6 \\
\hline Other sectors & LTOF & -74.6 & -107.4 & -96.9 & -48.0 & -53.6 & -48.6 & 11.5 & 90.5 & 22.9 & 1.8 & -49.2 \\
\hline Total & CGNG & -271.9 & -339.9 & -352.8 & -321.5 & -393.9 & -394.2 & -353.4 & -371.0 & -337.5 & -274.9 & -253.4 \\
\hline
\end{tabular}




\section{(0) 0 Reserve assets \\ ( - Central government sector}

Balance sheets valued at end of year

\begin{tabular}{|c|c|c|c|c|c|c|c|c|c|c|c|c|}
\hline & & 2000 & 2001 & 2002 & 2003 & 2004 & 2005 & 2006 & 2007 & 2008 & 2009 & 2010 \\
\hline Monetary gold & HCGD & 2.9 & 2.2 & 2.1 & 2.3 & 2.3 & 3.0 & 3.2 & 4.2 & 6.0 & 6.8 & 9.1 \\
\hline Special drawing rights & HCGE & 0.2 & 0.2 & 0.2 & 0.2 & 0.2 & 0.2 & 0.2 & 0.2 & 0.3 & 8.9 & 9.1 \\
\hline Reserve position in the Fund & HCGF & 2.9 & 3.5 & 3.8 & 3.5 & 2.9 & 1.0 & 0.7 & 0.5 & 1.6 & 2.1 & 3.2 \\
\hline \multicolumn{13}{|l|}{ Foreign exchange } \\
\hline With central banks & CGDE & 0.1 & 0.1 & 0.2 & 0.1 & 0.1 & 0.1 & 0.1 & 0.1 & 0.4 & 0.3 & 0.3 \\
\hline With other banks & CGDF & 3.7 & 2.8 & 1.9 & 1.3 & 0.3 & 0.8 & 0.4 & 0.4 & 0.4 & 0.3 & 0.3 \\
\hline Total currency and deposits & CGDD & 3.7 & 2.9 & 2.1 & 1.4 & 0.4 & 1.0 & 0.5 & 0.5 & 0.9 & 0.7 & 0.6 \\
\hline \multicolumn{13}{|l|}{ Securities } \\
\hline Bonds and notes & CGDH & 16.7 & 14.4 & 16.8 & 16.2 & 17.1 & 17.5 & 15.3 & 18.2 & 24.4 & 21.0 & 27.0 \\
\hline Money market instruments & CGDL & 2.3 & 2.2 & 0.2 & 0.2 & 0.3 & 1.7 & 3.0 & 3.1 & 3.1 & 0.7 & 0.6 \\
\hline Total securities & CGDG & 19.0 & 16.6 & 17.0 & 16.4 & 17.4 & 19.2 & 18.3 & 21.3 & 27.5 & 21.7 & 27.6 \\
\hline Total foreign exchange & HCGG & 22.7 & 19.4 & 19.1 & 17.7 & 17.8 & 20.2 & 18.8 & 21.7 & 28.4 & 22.3 & 28.2 \\
\hline Other claims & CGDM & 0.1 & 0.4 & 0.2 & - & 0.1 & 0.4 & - & 0.1 & - & - & 0.1 \\
\hline Total & LTEB & 28.8 & 25.6 & 25.5 & 23.8 & 23.2 & 24.7 & 22.9 & 26.7 & 36.3 & 40.1 & 49.7 \\
\hline
\end{tabular}




\section{External debt statement \\ End of period}

\begin{tabular}{|c|c|c|c|c|c|c|c|c|c|c|c|c|}
\hline & & 2000 & 2001 & 2002 & 2003 & 2004 & 2005 & 2006 & 2007 & 2008 & 2009 & 2010 \\
\hline \multicolumn{13}{|l|}{ General Government } \\
\hline \multicolumn{13}{|l|}{ Short-term } \\
\hline Money market instruments & HLYU & - & 0.1 & 0.2 & 1.9 & 3.8 & 2.8 & 3.5 & 7.2 & 21.0 & 19.4 & 27.9 \\
\hline Currency and deposits & HLVH & 0.1 & 0.1 & 0.1 & 0.1 & 0.1 & 0.1 & 0.2 & 0.2 & 0.2 & 0.1 & 0.1 \\
\hline Other liabilities & VTzz & 1.8 & 1.7 & 1.6 & 1.9 & 1.0 & 0.9 & 1.4 & 1.1 & 0.8 & 1.0 & 1.3 \\
\hline Total short-term & ZAVF & 2.0 & 1.9 & 1.9 & 3.9 & 4.9 & 3.8 & 5.1 & 8.5 & 22.0 & 20.6 & 29.4 \\
\hline \multicolumn{13}{|l|}{ Bonds and notes } \\
\hline issued by central government & HHGF & 62.4 & 59.9 & 56.4 & 66.1 & 83.8 & 110.7 & 135.5 & 160.2 & 203.6 & 224.3 & 309.2 \\
\hline \multicolumn{13}{|l|}{ Loans } \\
\hline to central government & HHGZ & 0.6 & 0.5 & 0.4 & 0.2 & 0.1 & 0.1 & - & - & - & - & - \\
\hline to local authorities & HHHA & 0.8 & 0.8 & 0.9 & 1.1 & 1.7 & 1.9 & 2.1 & 2.1 & 2.5 & 3.0 & 3.2 \\
\hline Total long-term & ZAVG & 63.7 & 61.2 & 57.6 & 67.4 & 85.7 & 112.6 & 137.6 & 162.2 & 206.1 & 227.3 & 312.4 \\
\hline Total General Government liabilities & ZAVH & 65.7 & 63.0 & 59.5 & 71.4 & 90.6 & 116.4 & 142.6 & 170.7 & 228.1 & 247.9 & 341.7 \\
\hline \multicolumn{13}{|l|}{ Monetary Authorities } \\
\hline \multicolumn{13}{|l|}{ Short-term } \\
\hline Money market instruments & VTzS & 0.2 & 1.4 & 2.8 & 3.5 & 3.4 & 3.6 & 2.4 & 2.5 & 3.3 & 2.6 & 2.6 \\
\hline Currency and deposits & VTZT & 5.2 & 3.8 & 5.5 & 6.8 & 9.9 & 13.0 & 14.6 & 21.6 & 36.8 & 13.8 & 17.3 \\
\hline Total short-term & VTZY & 5.5 & 5.2 & 8.3 & 10.3 & 13.3 & 16.5 & 16.9 & 24.0 & 40.2 & 16.4 & 20.0 \\
\hline \multicolumn{13}{|l|}{ Long-term } \\
\hline Bonds and notes & VTZU & - & - & - & - & - & - & - & - & - & - & - \\
\hline Total long-term & VTzV & - & - & - & - & - & - & - & - & - & - & - \\
\hline Total Monetary Authorities liabilities & VTZW & 5.5 & 5.2 & 8.3 & 10.3 & 13.3 & 16.5 & 16.9 & 24.0 & 40.2 & 16.4 & 20.0 \\
\hline \multicolumn{13}{|l|}{ Monetary financial institutions } \\
\hline \multicolumn{13}{|l|}{ Short-term } \\
\hline Money market instruments & ZAUX & 110.7 & 131.7 & 137.5 & 127.1 & 127.4 & 132.6 & 159.6 & 181.2 & 199.0 & 265.2 & 203.4 \\
\hline Currency and deposits & JXI3 & 1259.3 & 1369.0 & 1433.9 & 1598.5 & 1845.3 & 2186.8 & 2356.1 & 3121.9 & 3560.3 & 2962.5 & 3051.3 \\
\hline Total short-term & ZAVI & 1370.0 & 1500.7 & 1571.4 & 1725.6 & 1972.7 & 2319.4 & 2515.7 & 3303.1 & 3759.3 & 3227.7 & 3254.6 \\
\hline \multicolumn{13}{|l|}{ Long-term } \\
\hline Bonds and notes & HMBF & 77.4 & 84.1 & 95.0 & 121.7 & 154.0 & 201.3 & 234.1 & 306.2 & 358.4 & 388.8 & 383.7 \\
\hline Total long-term & ZPOK & 77.4 & 84.1 & 95.0 & 121.7 & 154.0 & 201.3 & 234.1 & 306.2 & 358.4 & 388.8 & 383.7 \\
\hline \multicolumn{13}{|l|}{ Total monetary financial } \\
\hline institutions liabilities & ZAVA & 1447.4 & 1584.8 & 1666.5 & 1847.3 & 2126.7 & 2520.7 & 2749.8 & 3609.3 & 4117.7 & 3616.5 & 3638.3 \\
\hline \multicolumn{13}{|l|}{ Other sectors } \\
\hline \multicolumn{13}{|l|}{ Short-term } \\
\hline Money market instruments & HLYQ & 21.7 & 22.5 & 30.6 & 23.7 & 22.5 & 22.2 & 16.3 & 19.1 & 29.9 & 21.5 & 25.4 \\
\hline Loans & ZLBY & 369.6 & 471.3 & 439.7 & 513.1 & 638.5 & 888.1 & 873.1 & 950.2 & 902.3 & 796.9 & 958.6 \\
\hline Trade credits & HCGB & 1.1 & 1.1 & 1.0 & 0.9 & 0.9 & 1.0 & 0.8 & 0.8 & 1.1 & 1.0 & 1.0 \\
\hline Other liabilities & LSYR & 1.1 & 1.6 & 1.0 & 1.0 & 0.9 & 1.0 & 1.1 & 1.3 & 1.5 & 1.6 & 2.4 \\
\hline Total short-term liabilities & ZAVB & 393.5 & 496.6 & 472.3 & 538.8 & 662.7 & 912.3 & 891.3 & 971.5 & 934.9 & 821.0 & 987.4 \\
\hline \multicolumn{13}{|l|}{ Long-term } \\
\hline Bond and notes & HHGJ & 121.3 & 129.5 & 160.1 & 211.7 & 258.3 & 329.3 & 371.3 & 433.4 & 559.4 & 630.0 & 626.4 \\
\hline Loans & ZLBZ & - & - & - & - & 0.4 & 0.4 & 0.4 & 0.4 & 0.4 & 0.4 & 0.4 \\
\hline Trade credits & HBWC & - & - & - & - & - & - & - & - & - & - & - \\
\hline Other liabilities & VTUF & 11.0 & 10.9 & 12.9 & 10.4 & 11.7 & 14.4 & 18.8 & 14.6 & 10.7 & 10.4 & 10.6 \\
\hline Total long-term liabilities & ZAUQ & 132.3 & 140.3 & 173.0 & 222.1 & 270.5 & 344.2 & 390.5 & 448.5 & 570.5 & 640.8 & 637.4 \\
\hline Total other sectors liabilities & ZAUR & 525.8 & 636.9 & 645.3 & 760.9 & 933.2 & 1256.5 & 1281.8 & 1420.0 & 1505.4 & 1461.8 & 1624.8 \\
\hline \multicolumn{13}{|l|}{ Direct investment } \\
\hline Debt liabilities to affiliated enterprises & HHDJ & 84.3 & 97.4 & 128.4 & 127.7 & 149.0 & 184.5 & 194.2 & 178.2 & 253.8 & 260.2 & 277.6 \\
\hline Debt liabilities to direct investors & HBVB & 123.2 & 160.0 & 173.0 & 168.9 & 176.5 & 202.5 & 221.2 & 193.5 & 212.0 & 205.4 & 203.9 \\
\hline Total liabilities to direct investors & ZAUY & 207.5 & 257.4 & 301.4 & 296.6 & 325.5 & 387.0 & 415.4 & 371.6 & 465.7 & 465.5 & 481.5 \\
\hline GROSS EXTERNAL DEBT & ZAUS & 2251.9 & 2547.4 & 2681.0 & 2986.5 & 3489.3 & 4297.1 & 4606.6 & 5595.6 & 6357.1 & 5808.2 & 6106.3 \\
\hline
\end{tabular}


Balance sheets valued at end of year

$£$ billion

\begin{tabular}{|c|c|c|c|c|c|c|c|c|c|c|c|c|}
\hline & & 2000 & 2001 & 2002 & 2003 & 2004 & 2005 & 2006 & 2007 & 2008 & 2009 & 2010 \\
\hline \multicolumn{13}{|l|}{ Financial derivatives assets } \\
\hline \multicolumn{13}{|l|}{ UK banks } \\
\hline Sterling & ZPNP & 49.8 & 43.5 & 56.7 & 44.1 & 46.1 & 51.3 & 62.9 & 84.2 & 180.2 & 112.0 & 157.6 \\
\hline Foreign currency & ZPNQ & 340.6 & 481.0 & 626.2 & 579.3 & 663.3 & 768.8 & 790.9 & 1294.0 & 3859.9 & 2064.4 & 2094.5 \\
\hline Total UK banks & ZPNA & 390.5 & 524.5 & 682.8 & 623.4 & 709.4 & 820.1 & 853.7 & 1378.1 & 4040.2 & 2176.4 & 2252.1 \\
\hline \multicolumn{13}{|l|}{$\begin{array}{l}\text { Other Financial Intermediaries } \\
\text { UK securities dealers }\end{array}$} \\
\hline Sterling & RUVI & 3.2 & 13.2 & 16.2 & 10.6 & 11.5 & 15.0 & 28.6 & 38.6 & 83.7 & 35.7 & .. \\
\hline Foreign currency & RUVJ & 52.6 & 51.9 & 70.9 & 144.0 & 104.7 & 186.5 & 234.5 & 400.6 & 849.4 & 456.9 & .. \\
\hline Total UK securities dealers & KN8A & 55.7 & 65.1 & 87.1 & 154.7 & 116.2 & 201.5 & 263.1 & 439.2 & 933.1 & 492.6 & 710.7 \\
\hline Other ${ }^{2}$ & D4AG & 0.5 & 0.6 & 0.7 & 1.1 & 0.4 & 0.5 & 0.2 & 1.9 & 2.6 & 2.6 & .. \\
\hline Total Other Financial Intermediaries & $\mathrm{D} 4 \mathrm{AH}$ & 56.2 & 65.7 & 87.8 & 155.8 & 116.6 & 202.1 & 263.3 & 441.1 & 935.7 & 495.2 & .. \\
\hline \multicolumn{13}{|l|}{ Insurance companies and pension funds } \\
\hline Insurance companies ${ }^{3}$ & $\mathrm{D} 4 \mathrm{AE}$ & 0.7 & 1.0 & 0.8 & 0.2 & - & - & - & 0.3 & 2.1 & 2.3 & .. \\
\hline Total insurance companies and pension funds & D4AF & 1.5 & 1.7 & 1.5 & 0.8 & 3.0 & 2.6 & 6.0 & 8.9 & 8.0 & 22.8 & .. \\
\hline Total UK assets & ZPNC & 448.2 & 592.0 & 772.1 & 780.0 & 828.9 & 1024.8 & 1123.0 & 1828.2 & 4983.8 & 2694.4 & \\
\hline
\end{tabular}

\section{Financial derivative liabilities}

UK banks
Sterling
Foreign currency
Total UK banks
Other Financial Intermediaries
UK securities dealers
$\quad$ Sterling
$\quad$ Foreign currency
Total UK securities dealers
Other
Total Other Financial Intermediaries
Insurance companies and pension funds
Insurance companies
Pension funds

Total UK liabilities

Net international investment position

Banks

Other Financial Intermediaries

Securities dealers

Other $^{2}$

Total Other Financial Intermediaries

Insurance companies and pension funds Insurance companies

Pension funds

Total insurance companies and pension funds

Total

\begin{tabular}{lrrrrrrrrrrr} 
ZPNR & 48.2 & 43.8 & 57.1 & 32.4 & 36.3 & 66.3 & 62.4 & 82.3 & 258.2 & 124.8 & 161.9 \\
ZPNS & 351.8 & 485.8 & 631.5 & 600.0 & 678.8 & 764.8 & 828.1 & 1309.9 & 3657.0 & 1972.0 & 2032.3 \\
\cline { 2 - 10 } ZPNB & 400.0 & 529.6 & 688.7 & 632.4 & 715.0 & 831.1 & 890.5 & 1392.2 & 3915.3 & 2096.8 & 2194.2
\end{tabular}

RUXE

RUXF

KN8B D4AK

D4AL

\begin{tabular}{rrrrrrrrrrr}
4.3 & 13.6 & 17.2 & 14.0 & 14.0 & 18.2 & 23.6 & 40.2 & 86.3 & 38.9 &.. \\
46.5 & 50.2 & 73.7 & 150.0 & 112.2 & 183.1 & 234.4 & 392.5 & 740.8 & 414.3 &.. \\
\hline 50.9 & 63.8 & 90.9 & 163.9 & 126.2 & 201.3 & 258.0 & 432.6 & 827.1 & 453.2 & 700.8 \\
0.1 & - & 0.1 & 0.7 & 0.1 & 0.1 & - & 1.8 & 2.2 & 3.2 &.. \\
\hline 51.0 & 63.8 & 91.0 & 164.6 & 126.3 & 201.4 & 258.0 & 434.5 & 829.3 & 456.4 &..
\end{tabular}

D4AI

GKGR

D4AJ

\begin{tabular}{rrrrrrrrrrr}
0.3 & 0.2 & 0.5 & 0.2 & 0.1 & - & - & 0.4 & 2.3 & 1.1 &.. \\
0.6 & 0.7 & 0.4 & 0.4 & 3.1 & 2.8 & 7.0 & 11.3 & 6.3 & 41.1 &.. \\
\hline 0.9 & 0.9 & 0.9 & 0.7 & 3.2 & 2.8 & 7.0 & 11.6 & 8.6 & 42.2 &..
\end{tabular}

ZPND

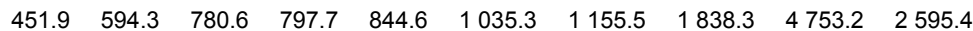

\begin{tabular}{|c|c|c|c|c|c|c|c|c|c|c|c|}
\hline ZPNE & -9.5 & -5.1 & -5.8 & -9.0 & -5.6 & -11.0 & -36.8 & -14.1 & 124.9 & 79.6 & 57.9 \\
\hline ZPNF & 4.9 & 1.3 & -3.8 & -9.2 & -10.0 & 0.2 & 5.1 & 6.6 & 106.0 & 39.4 & 10.0 \\
\hline D4AP & 0.3 & 0.6 & 0.6 & 0.4 & 0.3 & 0.4 & 0.1 & 0.1 & 0.4 & -0.6 & .. \\
\hline D4AQ & 5.2 & 1.9 & -3.2 & -8.8 & -9.8 & 0.6 & 5.3 & 6.7 & 106.4 & 38.8 & . \\
\hline $\mathrm{D} 4 \mathrm{AM}$ & 0.4 & 0.8 & 0.3 & - & -0.1 & -0.1 & - & -0.1 & -0.2 & 1.2 & . \\
\hline DAN & 0.2 & - & 0.3 & 0.1 & -0.1 & -0.1 & -1.0 & -2.6 & -0.5 & -20.6 & . \\
\hline D4AO & 0.6 & 0.8 & 0.6 & 0.1 & -0.3 & -0.2 & -1.0 & -2.7 & -0.7 & -19.4 & .. \\
\hline & -3.7 & -2.4 & -8.5 & -17.7 & -15.7 & -10.6 & -32.5 & -10.1 & 230.6 & 98.9 & \\
\hline
\end{tabular}

1 The data in this table for 'other financial intermediaries' other than securities 2 Includes unit and investment trusts and open-ended investment companies, dealers and for insurance companies and pension funds are developmental finance leasing companies, credit grantors, factoring companies and building and are not included in the main aggregates of the UK's international invest- societies.

ment position. However, the data in this table for UK banks have now been 3 Includes both general and long-term insurance.

included in the main aggregates of the international investment position from 4 Includes self-administered pension funds only. 2004 and the data for UK securites dealers have been included from 2010. 